\title{
Applications of neural networks to the studies of phase transitions of two-dimensional Potts models
}

\author{
C.-D. Li, D.-R. Tan, and F.-J. Jiang* \\ Department of Physics, National Taiwan Normal University, 88, Sec.4, Ting-Chou Rd., Taipei 116, Taiwan
}

\begin{abstract}
We study the phase transitions of two-dimensional (2D) $Q$-states Potts models on the square lattice, using the first principles Monte Carlo (MC) simulations as well as the techniques of neural networks (NN). We demonstrate that the ideas from NN can be adopted to study these considered phase transitions efficiently. In particular, even with a simple NN constructed in this investigation, we are able to obtain the relevant information of the nature of these phase transitions, namely whether they are first order or second order. Our results strengthen the potential applicability of machine learning in studying various states of matters. Subtlety of applying NN techniques to investigate many-body systems is briefly discussed as well.
\end{abstract}

\section{INTRODUCTION}

While originally being a topic in computer science such as the artificial intelligence, the techniques of machine learning (ML) have been applied to many other fields of science in the last a few decades 1 [ 8 . For example, the strategy of using methods of ML in analyzing large data sets of traffic flow and genome sequences has gained a lot of attention recently. Ideas from ML provide alternatives to the conventional approaches in various scientific areas. In some cases, the performance of these new approaches using the techniques of ML is as efficient as, or even better than the conventional ones. In the near future, it is anticipated that ML is likely to play a crucial role in many traditional fields of science.

The theoretical study of different phases of many-body systems, as well as the characters of the transitions between these various states is one of the major research topics in condensed matter physics ${ }^{9}$. Numerous numerical techniques, such as Monte Carlo simulations, series expansion, exact diagonalization, tensor networks and so on, are available for fulfilling these tasks $\frac{10 \mid 11}{1}$. Indeed, studies using these traditional methods have provided valuable information regarding the properties of matters. In particular, using the theoretical results obtained by these conventional techniques in conjunction with the data of relevant experiments, properties of some materials have been understood to an unprecedented precision.

With the advance of computing power as well as the development of ML algorithms, one interesting question arises, namely are these techniques of ML capable of efficiently studying different phases of matters? Such an idea has triggered pioneering works of applying ML algorithms to investigate phases, particularly the transitions between different states, of certain condensed matter systems $\sqrt{12}$. In these pioneering studies, it is demonstrated that the neural networks (NN) of ML is a powerful approach to distinguish phases of many-body systems. Furthermore, NN can even detect exotic states such as a phase with topological order. Finally, NN is capable of locating the place in the relevant parameter space where the transition between two phases occurs as well.

Motivated by the success of applying NN to study phases of condensed matter systems, in this investigation we examine if one can obtain further information regarding a phase transition beyond detecting the location of the critical point. For instance, it will be extremely interesting to determine whether a studied phase transition is continuous or not, by employing either the supervised or unsupervised NN techniques ${ }^{25}$. Notice for the studies of using supervised NN to investigate the phase transitions of condensed matter systems, the considered NN typically consists of one input layer, some hidden layers, including the convolutional and pooling layers, and one output layer as well as the weights connecting different layers. The constructed NN is then trained by feeding it with configurations of the two phases associated with the studied phase transition. Furthermore, in the training stage, a particular value (number) is assigned to each configuration as its default (and fixed) result in the output layer, and the weights are calculated by minimizing the so called loss functions during the training process. When the training is done, one can use the trained $\mathrm{NN}$ to obtained the outputs of the target configurations (called the testing set). These outputs are functions of the considered parameters and they provide us with relevant information regarding the critical points of the investigated phase transitions. For instance, it is shown convincingly that for the Ising model (on the square lattice), as the box sizes $L$ increase, the corresponding temperatures at which both the components of the output vectors are 0.5 converge to the correct critical temperature $e^{12}$.

While the outputs described in previous paragraph may be useful in determining whether the studied phase transitions are first order or second order, in this investigation we consider the possibility of using another powerful and completely different idea, implemented in the $\mathrm{NN}$ framework, to decide the nature of a phase transition. Specifically, in our study we use the histogram method which is one of the most frequently considered methods in the conventional approaches. The histogram method will be introduced briefly later in the related section. Remarkably, for the considered models here, namely the two-dimensional (2D) $Q$-states Potts models with $Q$ being a positive integer 26 , even a simple NN in conjunction with the histogram method can provide us with the 
relevant information to unambiguously determine the nature of the phase transitions, induced by tuning the temperatures, of these models. In particular, unlike the typical supervised NN approaches, in this study we confuse the built NN here by using only the configurations of the ordered phases as the training sets. As we will demonstrate later, this idea leads to efficient determination of the critical points in the relevant parameter spaces as well as the nature of the considered phase transitions. Our investigation is not only important and interesting in itself, the obtained results also strengthen the applicability of $\mathrm{NN}$ in studying condensed matter systems.

\section{MICROSCOPIC MODELS, OBSERVABLES, AND METHODS}

The Hamiltonian $H$ of the 2D $Q$-states Potts model on the square lattice considered in our study is given by

$$
\beta H=-\beta \sum_{\langle i j\rangle} \delta_{\sigma_{i}, \sigma_{j}}
$$

where $\beta$ is the inverse temperature and $\langle i j\rangle$ stands for the nearest neighboring sites $i$ and $j$. In addition, the Potts variable $\sigma_{i}$ appearing above at each site $i$ has the expression

$$
\sigma_{j}=\exp \left(i \frac{2 \pi s_{j}}{Q}\right), s_{j}=1,2,3, \ldots, Q .
$$

In the literature, for the $2 \mathrm{D} Q$-states Potts models on the square lattice, the phase transitions due to fluctuation in temperatures are second order for $Q \leq 4$, and first order when $Q \geq 5$. In this investigation, we have studied these phase transitions using both the method of Monte Carlo (MC) calculations and the techniques of NN. In particular, special attention has been focused on whether the methods of NN can be employed to determine the nature of these considered phase transitions efficiently.

To study these phase transitions through the MC simulations, the observable magnetization per site $\langle|m|\rangle$, where $m$ takes the following form

$$
m=\frac{1}{L^{2}} \sum_{i} \sigma_{i}
$$

is measured. Here $L$ is the linear box sizes used in the simulations. Besides, the energy density (energy per site) $\langle E\rangle$ is calculated as well. By studying the histograms of these two physical quantities, the nature of these phase transitions, i.e., first order or second order, can be effectively decided.

In addition to the conventional approach of using $\mathrm{MC}$ simulations, we also investigate these phase transitions with the techniques of supervised NN. By considering quantities which are defined within the NN framework, we find that the nature of these phase transitions can be accurately determined as well. The procedures, including the considered quantities, associated with the constructed NN in this study will be described in details in the relevant sections.

\section{THE NUMERICAL RESULTS}

To investigate the nature of the considered phase transitions, we have performed $\mathrm{MC}$ simulations for $Q=2,3,4,5$, and 10, using the Swendsen Wang algorithm 29 . In particular, at every considered temperature close to the critical temperatures $T_{c}(Q, L)$, a few thousand configurations are stored for each studied $Q$ and $L$. These configurations will be used in the NN approach to construct the appropriate quantities serving as the main information of deciding the nature of the considered phase transitions. In the next subsection, we will briefly present the numerical results obtained from the MC simulations.

\section{A. The results of MC Simulations}

One efficient method to determine the nature of a phase transition, namely to decide whether the transition is first order or second order, is to investigate the histograms of some relevant observables. Specifically, if a phase transition is first order, then for sufficiently large $L$, two peaks will appear in the histograms of certain observables when the simulations are carried out at (or close to) the corresponding critical point. Furthermore, the relative heights between the peak $(\mathrm{s})$ and the trough (located in the middle) will approach infinity as one increases $L$. The appearance of the two peaks scenario for a first order phase transition is due to the fact that at the critical point the system is in either phase with equal probabilities. On the other hand, if the considered phase transition is second order, then the related histograms do not have these features. It should be pointed out that while for a second order phase transition, one may observe the appearance of two peaks in the histograms (of certain observables), the mentioned relative heights between the peak(s) and the trough will saturate to a constant. With this powerful idea, we indeed find that while the phase transitions for 2D Potts models on the square lattice with $Q=2,3$, and 4 are continuous, the corresponding transitions of $Q=5$ and 10 are first order.

The histograms of $\langle|m|\rangle$ for 2-states Potts models determined with $L=40$ are shown in fig. $1_{1}^{\mathrm{B} 2}$. In particular, the results in fig. 1 are obtained at temperatures 1.133 (top) and 1.260 (bottom). We find that no two peaks structure shows up for other temperatures, separating from each other by 0.001 , between $T=1.133$ and $T=1.260$. We have additionally simulated $L=80$ lattices for 2-states Potts model and have found a similar scenario as that of $L=40$. Based on these outcomes, 

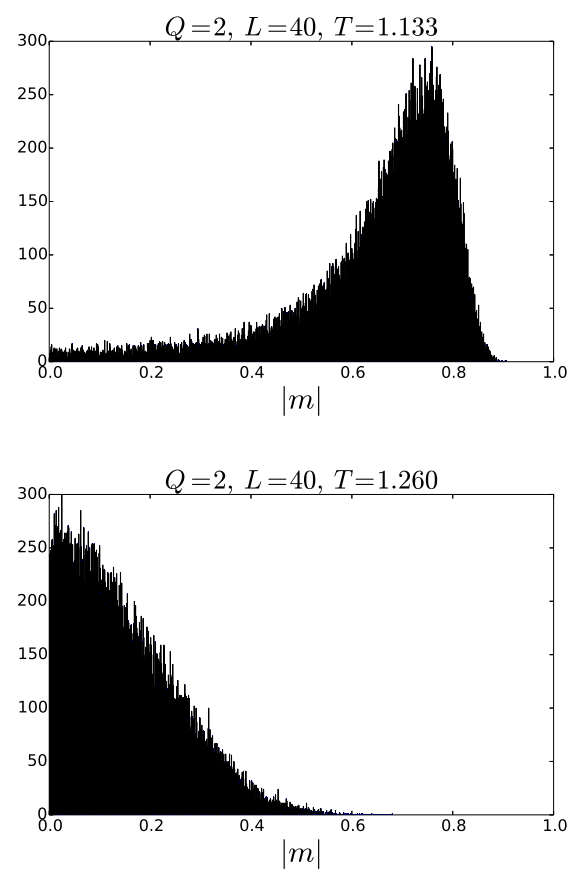

FIG. 1. Histograms of $\langle|m|\rangle$ at $T=1.133$ (top) and $T=1.260$ (bottom) for 2D 2-states Potts model with $L=40$ on the square lattice. Several ten thousand data, separating by few thousand updates from each other after the thermalization, are generated for each of the plots. The histograms are produced by the "hist" function of pylab 33 .

one concludes that the phase transition of 2-states Potts model is second order.

The histograms of $\langle|m|\rangle$ for 10-states Potts models, determined at $L=20$ and $L=40$, are demonstrated in fig. 2. Notice two peaks clearly appear for both $L=20$ and $L=40$ in fig. 2 . In particular, this two peaks structure is more noticeable for $L=40$. These results indicate that the phase transition for 10-states Potts model is indeed first order.

To summarize, figs. 1 and 2 clearly show convincing signals that the phase transitions of 2- and 10-states Potts models are second order and first order, respectively. It should be pointed out that because $Q=4$ and $Q=5$ are at the edges of separating second and first order phase transitions of 2D Potts models on the square lattice, data of larger $L$ are needed in order to reach a conclusive answer of whether the phase transitions of 4- and 5-states Potts models are second order or first order. Although our data for 4- and 5-states Potts model do not lead to absolutely conclusive results, they do show the correct trends.

\section{B. The Results of NN Methods}

Besides the conventional methods, as the one of Monte Carlo simulations shown in the previous subsection, in the following we will employ the techniques of supervised
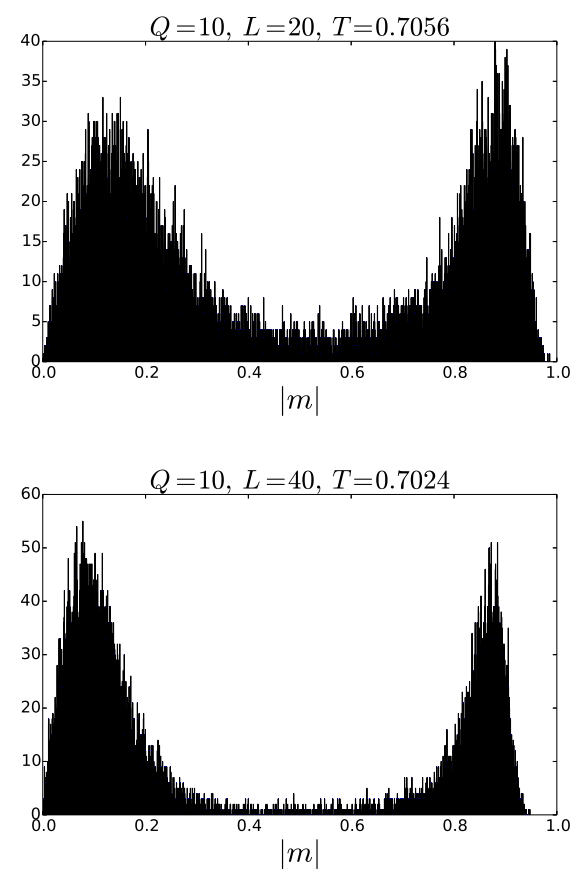

FIG. 2. Histograms of $\langle|m|\rangle$ for $L=20$ (top) and 40 (bottom) for $2 \mathrm{D} 10$-states Potts model on the square lattice. Around twenty thousand data, separating by few thousand updates from each other after the thermalization, are generated for each of the plots. The histograms are produced by the "hist" function of pylab 33 .

ML to study the considered phase transitions.

\section{The training sets, labels, and outputs of $N N$}

Before presenting the numerical results obtained using the techniques of supervised ML, we will firstly detail the ideas behind our NN approach. In particular, the considered NN training sets and labels will be introduced thoroughly. To begin with, a typical NN procedure of studying the phase transition of Ising model will be described briefly.

When one investigates the phase transition of Ising model with the supervised NN techniques, the training set consists of configurations obtained by Monte Carlo simulations performed over a broad range of temperatures. Furthermore, these selected temperatures should be from both sides of the critical temperature $T_{c}$. The label of a configuration in the training set is an assigned two-component vector, taking the result of either $(1,0)$ or $(0,1)$. These vectors, namely $(1,0)$ and $(0,1)$, serve as the flags to indicate whether the configurations are in the disordered phase or the ordered phase. Notice the training is conducted for every considered lattice with linear size $L$. After the completion of training, one then uses the trained NN to calculate the outputs of many testing configurations obtained with temperatures cross$\operatorname{ing} T_{c}$. In this testing stage, for each linear lattice size 


$$
\begin{aligned}
& \text { pre-training set } \\
& \left(\begin{array}{cccc}
1 & 1 & \cdots & 1 \\
1 & 1 & \cdots & 1 \\
\vdots & \vdots & \cdots & \vdots \\
1 & 1 & \cdots & 1
\end{array}\right) \longrightarrow \\
& \left(\begin{array}{cccc}
2 & 2 & \cdots & 2 \\
2 & 2 & \cdots & 2 \\
\vdots & \vdots & \cdots & \vdots \\
2 & 2 & \cdots & 2
\end{array}\right) \longrightarrow\left(\begin{array}{l}
1 \\
0 \\
0
\end{array}\right) \\
& \left(\begin{array}{cccc}
3 & 3 & \cdots & 3 \\
3 & 3 & \cdots & 3 \\
\vdots & \vdots & \cdots & \vdots \\
3 & 3 & \cdots & 3
\end{array}\right) \longrightarrow\left(\begin{array}{l}
0 \\
1 \\
0
\end{array}\right)
\end{aligned}
$$

FIG. 3. The pre-training set and their corresponding NN labels for 3-states Potts model. Each possible ground state configuration on a $L$ by $L$ lattice is represented by a $L$ by $L$ matrix. In particular, the matrix entries are the values of the Potts variables at the corresponding lattice sites. Such a correspondence between the (ground state) configurations in the pre-training set and their labels can be applied to other $Q$-states Potts models in exactly the same manner.

$L$, the corresponding critical temperature $T_{c}(L)$ is the temperature at which the output vector is $(0.5,0.5)$.

Having briefly introduced a typical procedure of studying the phase transition of Ising model using the NN methods, in the following we will specify our NN approach to the investigation of $Q$-states Potts models. In particular, the ideas behind the constructed $\mathrm{NN}$ used in our study will be explained in detail. Notice for $Q$-states Potts model on a $L$ by $L$ lattice, one intuitively expects that the corresponding ground states consist of $Q$ configurations. In addition, for the $j$ th ground state configuration, the associated Potts variable at every site has the same positive integer value $j$. On the other hand, at temperatures $T$ much higher than the critical temperature $T_{c}$, the corresponding Potts variable at each site of a $L$ by $L$ lattice can take any number $n_{2}$ in $\{1,2,3, \ldots, Q\}$ with equal probability. These two propositions are confirmed by the snapshots of our Monte Carlo simulations. Based on these results, instead of performing our study following the related standard NN procedures, here we consider a completely different approach which is much simpler conceptionally. Specifically, in our NN calculations, for any $Q$-states Potts model we use the associated $Q$ (ground state) configurations as the objects in the pretraining set (We will explain later why we call these configurations the pre-training set). In other words, unlike the typical NN approaches, the training sets considered here are not generated numerically by Monte Carlo simulations. On the contrary, they are created by hand and are the expected ground state configurations of the studied models.

Notice for the Ising model, if one uses ordered and dis- ordered states as the two categories (classifications) for the training set, then the corresponding NN labels are two-component vectors. In particular, during the training stage the labels for the ordered and disordered states are $(1,0)$ and $(0,1)$ (or vice versa), respectively. Inspired by this, we consider $Q$-vectors as the NN labels (and outputs) for $Q$-states Potts model. Specifically, in the training process, a (ground state) configuration on a $L$ by $L$ lattice with each Potts variable taking the same integer value $n_{1}$ is assigned a $Q$-vector as its label. In addition, this $Q$-vector has the property that all its components are 0 except the $n_{1}$ th component which is 1 . Figure 3 shows the connection between the objects in the pre-training set and their corresponding labels, namely $Q$-vectors for 3 -states Potts model. The $L$ by $L$ matrices of the left hand side (LHS) panel of fig. 3 represent the configurations in the pre-training set, namely the three ground state configurations of 3 -states Potts model on a $L$ by $L$ lattice. In particular, each matrix entry stands for the Potts variable at the corresponding lattice site. Finally it should be pointed out that the output layer in our NN, which will be introduced later, is activated by a softmax function. As a result, the summation of all components of any output $Q$-vector is 1 .

One may wonder whether the NN with such purposely designed pre-training sets and labels is capable of investigating the phase transitions of the considered models efficiently. In the following, we will argue theoretically that the NN with the pre-training sets and labels introduced in the previous paragraph can be used as a valid and effective tool for uncovering the physics of the considered phase transitions.

As described previously that for any $Q$-states Potts model with a given positive integer $Q$, the considered pre-training set consists of $Q$ objects which are the associated ground state configurations. Furthermore, for the $n$th object in the pre-training set, all its Potts variables take the same positive integer value $n$. The labels for the objects in the pre-training set are $Q$-vectors and the correspondence between a training configuration and its label, i.e. a $Q$-vector, has already been defined earlier and can be understood pictorially by fig 3 . Notice as one approaches the critical temperature $T_{c}$ from very low temperatures, any Potts variable of a configuration obtained by the Monte Carlo simulations may begin to take a different value from those of other Potts variables of that configuration. The closer to the $T_{c}$, the distribution of the values of the Potts variables are more random. For configurations determined above $T_{c}$, the Potts variables take their possible results in a totally random manner over the whole lattices.

The scenario of how the distribution of the values of the Potts variables changes from low temperatures to high temperatures will reflect in the NN output $Q$-vectors as follows. When one supplies the already trained NN with a configuration obtained at a temperature extremely far below $T_{c}$, one component (could be any of the $Q$ components) of the output $Q$-vector has a numerical value much 
larger than those of other components and the difference could be several order in magnitude. Such a result described above occurs because most of the Potts variables of the configuration take the same positive integer value. In addition, if one (testing) configuration is determined with a relatively lower temperature than $T_{c}$, besides the fact that the majority of the Potts variables take one particular $i$ in $\{1,2,3, \ldots, Q\}$ as their value, theoretically the remaining part of the Potts variables will result in any allowed positive integer other than $i$ with a more or less equal amount of each. Consequently, the resulting output $Q$-vector has the following characteristics. Specifically, in addition to the fact that one component has the largest number $d$, other components of that $Q$-vector will begin to take non-negligible values, although these values may be relatively smaller than $d$. Finally if the values of the Potts variables of a testing configuration are randomly distributed all over the sites, then to the trained NN every one of the $Q$ ground states is the equally possible candidate for the testing configuration. For such a case, which corresponds to the situation that the simulated temperature $T$ is relative higher than $T_{c}$, each component of the resulting $Q$-vectors has a value around $1 / Q$. Based on these observations described above, as one moves from temperatures below $T_{c}$ to those above $T_{c}$, every component of the related output $Q$-vectors is approaching the same value $1 / Q$.

As we have already described earlier, for testing configurations determined with the same box size $L$ and the same temperature $T$ (which is relatively lower than $T_{c}$ ), although the output $Q$-vector may be different component-wisely from one configuration to another configuration, for majority of these testing configurations the norms of the related output $Q$-vectors should be approximately the same ideally. This is because every possible integer for the Potts variables has equal footing with each other. As a result, the norm $R$ of the output $Q$-vectors will be a suitable (and better) quantity for our investigation. For testing set determined by Monte Carlo simulations with extremely low temperatures, the corresponding outcomes of $R$ are 1 . As one moves from low temperatures toward the vicinity of $T_{c}$, the norms of the NN output $Q$-vectors change from 1 to around $1 / \sqrt{Q}$. For $T>T_{c}$, the resulting $R$ will be $1 / \sqrt{Q}$ theoretically. In other words, the quantity $R$, namely the norm of the NN output $Q$-vectors can be used as an effective observable to study the phase transitions of the considered $Q$-states Potts models. Furthermore, as we will demonstrate later, $R$ is not only a valid quantity for estimating the critical temperatures, but is also an effective observable of determining the nature, namely whether they are first order or second order, of the considered phase transitions.

\section{The construction of $N N$}

After detailing the ideas behind the training sets and the corresponding labels used here, in the following we will introduce the NN considered in our study. In particular, we want to build a simple (deep learning) NN that enables us to carry out the related investigation. Notice the Keras library $\sqrt{34}$ is employed in the NN construction. In addition, the set up of the NN in this study consists of one input layer, one convolutional layer with kernel size 3 by 3 , one average pooling layer with kernel size 2 by 2 , and one output layer. The initial matrix elements (weights) $W_{i j}$ connecting to the output layer, as well as the pooling kernel are the default ones of Keras.

Notice that in our original proposition, for a given $Q$ states Potts model the training set, or more accurately the pre-training set contains $Q$ types of ground state configurations which may be not sufficient for a successful training. Indeed, in a typical study of a phase transition using the NN methods, depending on the batch size used in the training process, several hundred to (a) few thousand training configurations may be needed in order to ensure the success of the training. One of the reasons of considering sufficiently many training objects is to make sure that the trained NN is capable of classifying any validation and testing configurations with high precision. Furthermore, during the minimization procedures, the strategy of using enough amount of training objects also helps in reaching good results for the desired weights connecting different layers (Of course, one has to pay attention to some subtle issues such as overfitting as well). Therefore, in our investigation multiple copies of the original $Q$ types ground state configurations are used as the actual training set. This is the reason why the $Q$ types ground state configurations are called the pre-training set in this study. Notice in our NN procedures, after the step of one-hot encoding, a random shuffle among the training objects is performed (Such an operation can be one of the standard steps for the training of a NN). As a result, the training objects contained in any batch may be significantly different from those of other batches. In addition, with the strategy of carrying out a random shuffle on the training objects, if one performs two training processes, then the initial objects in each (ordered) batch of these two training processes will be different as well. Therefore, technically speaking, while originally one has $Q$ configurations in the pre-training set, the use of multiple copies of the $Q$ types ground state configurations as the actual objects in the training set does practically enlarge the space of the pre-training set.

We find that when $N \geq(n \times$ batch size $)$ copies of the ground state configurations are used as the training set, the obtained NN results are stable. Here the suitable value of $n$, which is a positive integer, requires some trial investigation. In this study the outcomes determined with $N=200$ (and the used batch size is 20) are shown explicitly. It should be pointed out as well that the ratio between $N$ and the used batch size cannot be too large. This is because when this ratio is too large, the minimization procedures will be unstable due to the facts that each of many batches may contain the same type of training objects in itself, or numerous batches could 
have almost-identical (or identical) training items among themselves. Finally we would like to emphasize the fact again that in our calculations, a configuration on a $L$ by $L$ lattice is represented by a $L$ by $L$ matrix. Furthermore, the matrix elements are the values of the Potts variables located at the associated sites. The details of the training for our constructed NN for any $Q$-states Potts model is as follows.

For a given fixed box size $L$ and for each number $i$ in $\{1,2,3, \ldots, Q\}, 200$ identical $L \times L$-matrices are created. In particular, every element in these matrices has an initial numerical value $i$. This procedure leads to $200 \times Q$ objects. These $200 \times Q$ objects are then one-hot encoded, resulting in yet another $200 \times Q$ objects for which each of them consists of $Q$ layers of $L \times L$-matrices. These $200 \times Q$ objects are the training set for the set up NN. The procedure of creating the training sets from the pretraining configurations is depicted in the sub-fig. $(a)$ of fig. 4 .

The convolutional layer is activated by rectified linear functions (ReLUs), see the $f$ at the bottom of sub-fig. (b) of fig. 4 Notice in the sub-fig. (b) of fig. $4 \quad \tilde{a}$ are the outcomes of applying the ReLUs to the results after the convolutional layer. We have considered different initial filter kernels, and find that there are some restrictions on the corresponding kernel matrix elements. Specifically, each matrix element should not be too large, otherwise one hardly obtains satisfactory results. In addition to the input layer, the convolutional layer, and the output layer, the built NN contains an average pooling layer as well (sub-fig. (c) of fig. 44). The full pictorial representation of the set up $\mathrm{NN}$ is depicted in fig. 4 .

The main algorithm considered in the built $\mathrm{NN}$ is the mini-batch. As a result, the $200 \times Q$ objects of the training sets will be permuted randomly before the actual training is conducted. Furthermore, we exploit the Adam optimizer of Keras to update the weights of the constructed NN. For the loss functions we use "categorical crossentropy" which is defined as

$$
C=-\frac{1}{n} \sum_{x} \sum_{j}^{Q}\left[y_{j} \ln a_{j}+\left(1-y_{j}\right) \ln \left(1-a_{j}\right)\right],
$$

where $n$ is the total number of objects in the training set, $a_{j}$ are the outcomes obtained after applying all the constructed layers. In addition, $x$ and $y$ are training inputs and the corresponding designed outputs, respectively. Finally, in our study we use the default $\eta$ as the learning rate.

In our investigation, as we have already mentioned earlier that various batch sizes, ranging from 10 to 80, are used to examine the stability of the output results with respect to the amount of copies of the pre-training set used as the objects in the training set. In addition, the number of epoch is confined by an early stop criterion and $L 2$-regularization is imposed in calculating the loss functions. The output layer in the set up NN is activated by a softmax function. As a result, the sum of all com-
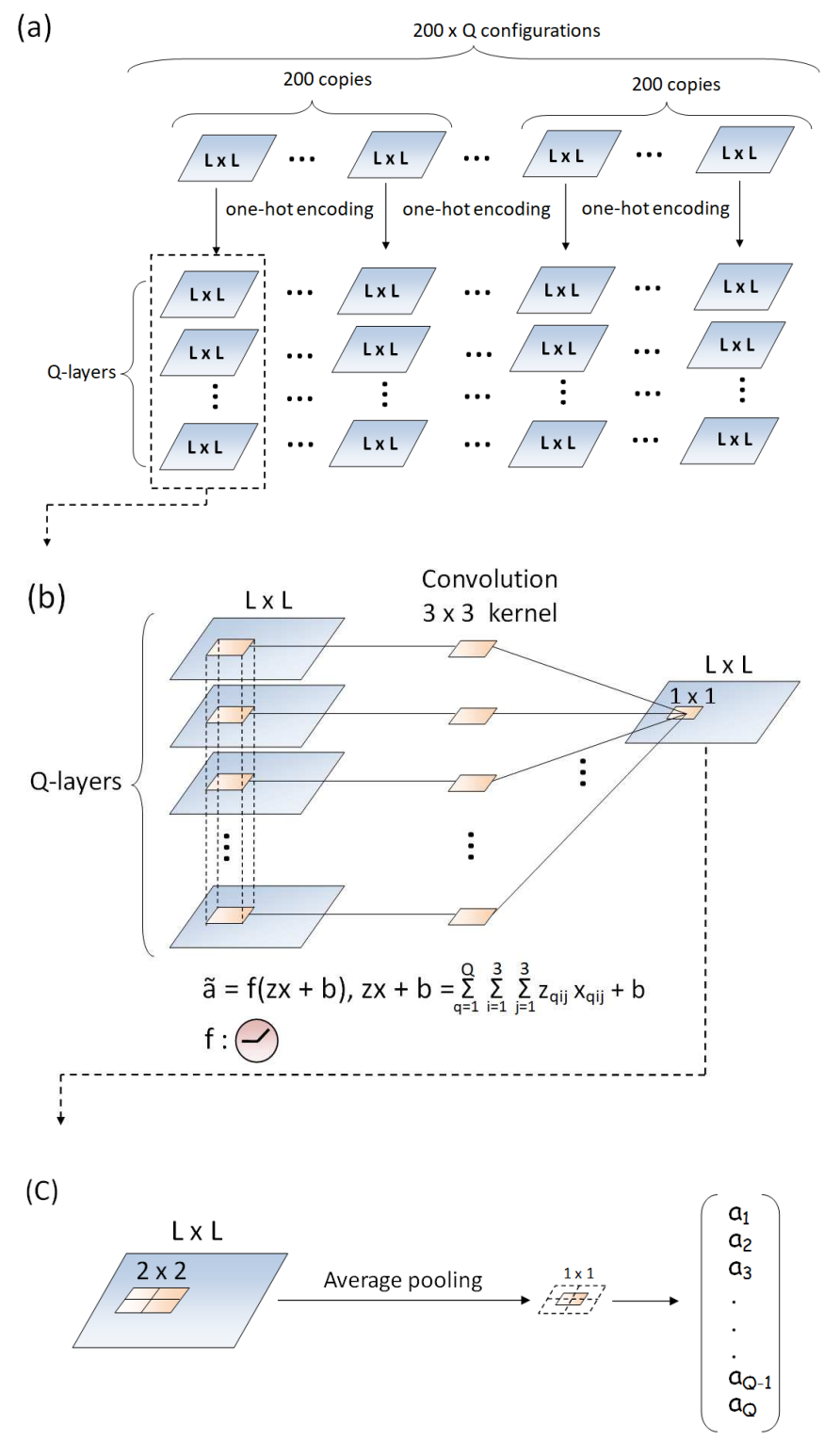

FIG. 4. The set up NN in this study. The weights connecting different layers are not shown explicitly. While panel (a) demonstrates the preparation of training set, panels (b) and (c) show how the convolution and average pooling work on one of the $200 \times Q$ training objects, respectively. In the actual calculations, results from all the $200 \times Q$ training objects are taken into account. Notice after the step of one-hot encoding, a random shuffle among the $200 \times Q$ objects is conducted.

ponents of a output vector is 1 . Notice there are several tunable parameters in our $\mathrm{NN}$ and we will make a remark regarding this later.

\section{The validation and testing of the constructed $N N$}

When the training of a built NN is done, one has to carry out the validation process to ensure the success of 
the training, namely to confirm the trained NN is capable of recognize every input from the validation set with high accuracy. For $Q$-states Potts models, we use 100 copies of the corresponding $Q$ ground state configurations as the validation sets. Furthermore, we find that the accuracy of the validation for the NN used here is 100 percent. It should be pointed out that while a random permutation is performed among the objects in the training sets before the actual training is executed, the training and validation sets considered in our study are actually the same from a general point of view. As a result, the validation conducted here should be viewed as a guidance for the success of the training stage. Whether the constructed $\mathrm{NN}$ can fulfill the desired tasks of determining the critical points as well as uncovering the nature of the studied phase transitions, should be evaluated based on if the NN results are consistent with the established outcomes obtained by the traditional methods.

For each temperature, the testing set for the constructed NN consists of several thousand configurations generated by $\mathrm{MC}$ simulations (separating from each other by few thousand updates). Notice with the built NN, as we already explained earlier, at the temperatures (far) below $T_{c}$, ideally one will find that each component of the output vector for every configuration generated by Monte Carlo simulations is zero, except one component which takes the value of 1 . The norms of such vectors are 1 . On the other hand, for the temperatures above $T_{c}$, since the values of Potts variables are in a totally random manner over all the lattice sites, the trained NN is not able to definitely decide which of the $Q$ possible (ground) states the system belongs to. Notice for such cases, every Potts variable will take any of the integers in $\{1,2,, . ., Q\}$ with equal probabilities. Therefore it is expected that each component of the corresponding output vectors has $1 / Q$ as its value theoretically (The norms of these vectors are $1 / \sqrt{Q}$ ). Based on these observations, similar to the conventional approaches, investigating the norms and their histograms of the output $Q$-vectors may shed some light on locating the critical temperatures as well as uncovering the nature of these phase transitions. In particular, for any given $Q$-states Potts model, the associated critical temperature $T_{c}$ should lie within the temperature interval $\left(T_{1}, T_{2}\right)$, where $T_{1}$ and $T_{2}$ are the temperatures at which $R$ begins to drop from its upper bound 1 and reaches its lower bound $1 / \sqrt{Q}$ for the first time, respectively.

Remarkably, even with the simple training sets (and labels) considered here, the constructed NN can be used to accurately estimate the critical temperatures $T_{c}$ of the investigated phase transitions. The norms $R$ of the NN output vectors as functions of $T$ for 2D 3-, 4-, 5- and 10states Potts models are shown in fig. 5 . We find that for each of the studied models, as $L$ increases, the range of $T$ for which $R$ drops from 1 to $1 / \sqrt{Q}$ converges quickly to a narrow region. In particular, the known critical temperatures in the literature for the investigated phase transitions lie within these regions of $T$. For example, the
$T_{c}$ for 3-states Potts model is given by $T_{c} \sim 0.99497$ in the literature, and one clearly sees from the most top panel of fig. 5 that as the box size $L$ increases, the range of temperatures where $R$ drops from 1 and saturates to $1 / \sqrt{3}$ converges to a narrow region containing the expected $T_{c} \sim 0.99497$.

\section{The nature of the considered phase transitions}

Notice while it is beyond doubt that the built NN in this study can precisely determine the $T_{c}$ of the considered phase transitions, fig. 5 does not provide us with sufficient information to decide whether they are first order or second order. To uncover the nature of these phase transitions of 2D Potts models using the constructed NN shown in fig. 4, we turn to studying the histograms of $R$. This strategy is motivated by one of the traditional methods, as shown previously in the subsection of Monte Carlo results. Notice since for temperatures which are relatively far below and above $T_{c}$, the numerical values of $R$ are given by 1 and $1 / \sqrt{Q}$, respectively, in the histogram(s) of $R$ one may observe two peaks located at $R=1$ and $R=1 / \sqrt{Q}$ near $T_{c}$. In particular, for a first order phase transition the relative heights between the peak $(\mathrm{s})$ and the trough should increase with the linear box sizes $L$. Here we would like to emphasize again the fact that for a first order phase transition, the appearance of two peak phenomena in the histograms of certain observables is because the system is equally probable in either one of two possible states. For our case, one of these two probable states has $R=1$ and the other has $R=1 / \sqrt{Q}$. Therefore, studying the histograms of $R$ may shed some light on uncovering the nature of the investigated phase transitions.

Remarkably, such a scenario introduced in the previous paragraph does happen in the histograms of $R$ for 2D 10states Potts model. The results depicted in fig. 6 are the histograms of $R$, obtained with $L=10,20$ and 80 close to their corresponding critical temperatures $\left(T_{c}(L)\right)$, for $2 \mathrm{D}$ 10-states Potts model. Fig. 6 shows that two peaks phenomena appear for all three lattices, and this phenomena becomes more and more noticeable as one increases the linear lattice size $L$. In particular, almost all the obtained results of $R$ for $L=80$ are located at $R=1.0$ and $R=1 / \sqrt{10} \sim 0.31623$. This implies that the phase transition of 2D 10-states Potts model is first order as expected. Notice the temperatures at which this two peaks phenomenon is the most obvious may be slightly different from those shown in fig. 2. This small discrepancy can be attributed to the fact that $T_{c}(L)$ are observable dependence.

Figures 7 and 8 show the histograms of $R$ close to $T_{c}$ for $2 \mathrm{D} 3$-states Potts models. From these figures we find that as one moves from low temperatures to high temperatures, the histograms of $R$ for 2D 3-states Potts model evolve from a configuration with (only) one peak locating near $R=1$ to a configuration with (only) one 

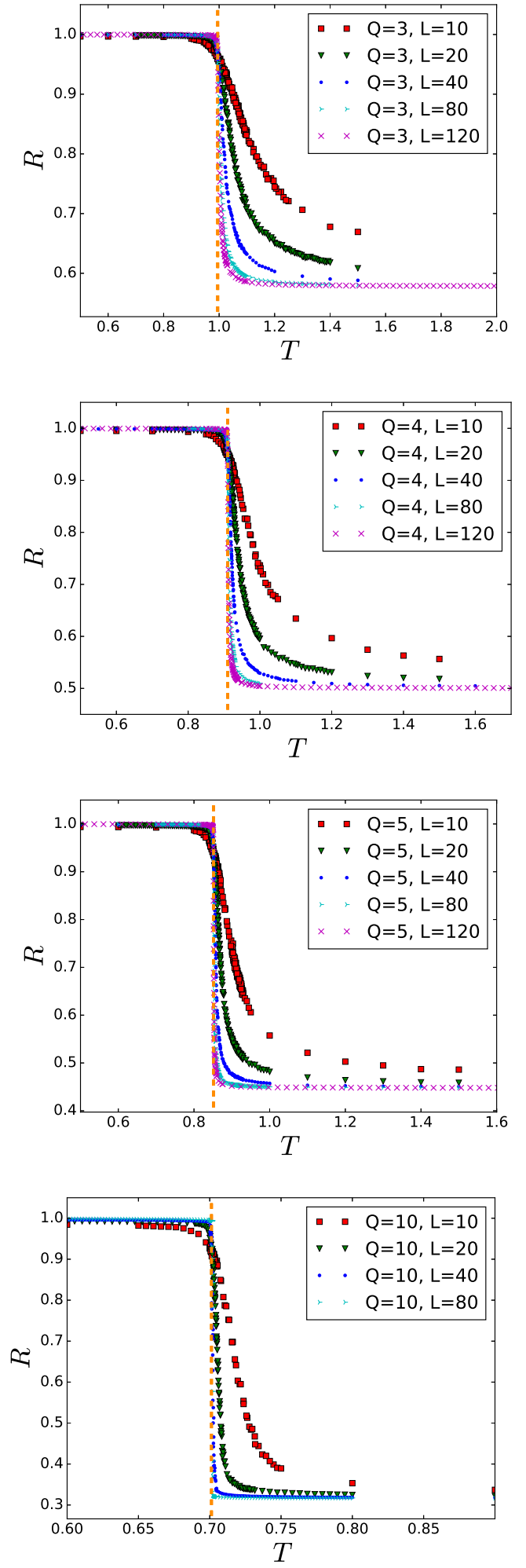

FIG. 5. (From top to bottom) The norms $R$ of the NN output vectors as functions of $T$ for 3-, 4-, 5-, and 10-states Potts models (and for several linear lattice sizes $L$ ). For comparison (and better visualization as well), in each panel a vertical dashed line which intersects the $T$-axis at the known $T_{c}$ in the literature is added by hand.
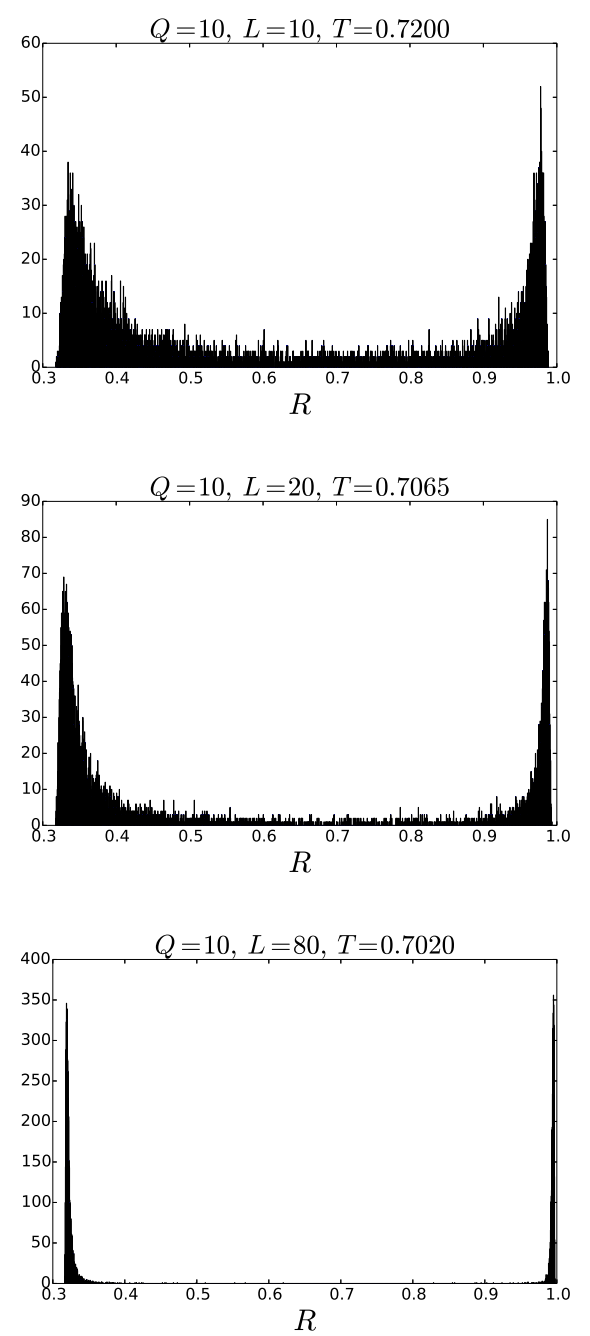

FIG. 6. Histograms of $R$ for $L=10$ (top), 20 (middle), and 80 (bottom) for 2D 10-states Potts model. Around ten thousand data points close to the corresponding $T_{c}$ are generated for each of the plots. Data are recorded once for every 2000 updates after the thermalization. The histograms are produced by the "hist" function of pylab 33 .

peak near $R=\frac{1}{\sqrt{3}}$, without two peaks structure like those of figure 6 appearing for the intermediate temperatures. From this observation as well as the fact that in figure 8 the relative heights between the peaks and trough are almost the same for $L=20,40$, and 240 , one concludes that the corresponding phase transition is second order. These two results of 2D 3- and 10-states Potts models obtained using the constructed $\mathrm{NN}$ in this study agree with those determined by other traditional methods.

Notice for 10-states Potts model, the temperature at which the two peaks structure is the most obvious for $L=80$ is around 0.702 which agrees very well with the expected $T_{c} \sim 0.70123$. In addition, for $2 \mathrm{D} 3$-states Potts model, the temperature at which the histogram of $R$ for $L=240$ is the most flat is around $T \sim 0.99775$. The temperature $T \sim 0.99775$ again matches nicely with the theoretical prediction $T_{c} \sim 0.99497$. These results pro- 

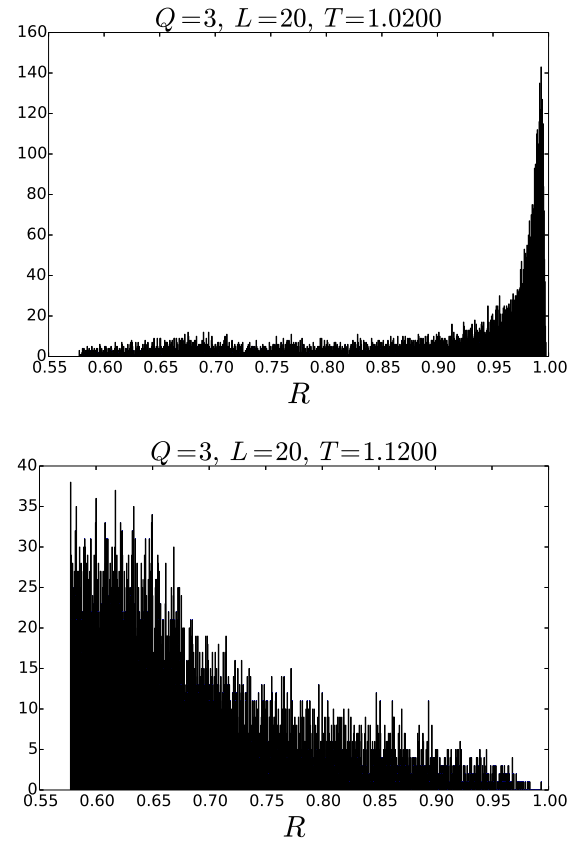

FIG. 7. Histograms of $R$ for $L=20$ at temperatures $T=1.02$ (top) and $T=1.12$ (bottom) for 2D 3-states Potts model. Around ten thousand data are generated for each of the plots. Data are recorded once for every 2000 updates after the thermalization. The histograms are produced by the "hist" function of pylab 33 .

vide convincing evidence that the NN constructed based on our ideas is able to locate the critical points of the considered models in the relevant parameter spaces. Furthermore, it is capable of detecting the nature of the studied phase transitions efficiently as well. Finally, by studying the histograms of $R$ for 2D 2-,4-,and 5-states Potts models, we also arrive at conclusions consistent with the known results in the literature regarding the nature of these phase transitions.

\section{DISCUSSIONS AND CONCLUSIONS}

In this study, we investigate the phase transitions of various $2 \mathrm{D} Q$-states Potts models using both the $\mathrm{MC}$ simulations and the techniques of NN. Special attention has been focused on whether the NN methods can be applied efficiently to determine the nature of the considered phase transitions. Remarkably, while one expects MC simulations is capable of carrying out such tasks, it is surprising that the simple NN constructed here provides us with clear signals to decide whether these phase transitions are first order or second order. Many pioneering works have demonstrated that $\mathrm{NN}$ is a powerful tool to distinguish phases of matters, and the results shown here certainly strengthen the applicability of NN in investigating condensed matter systems. One natural next step will be to build a NN capable of accurately calcu-
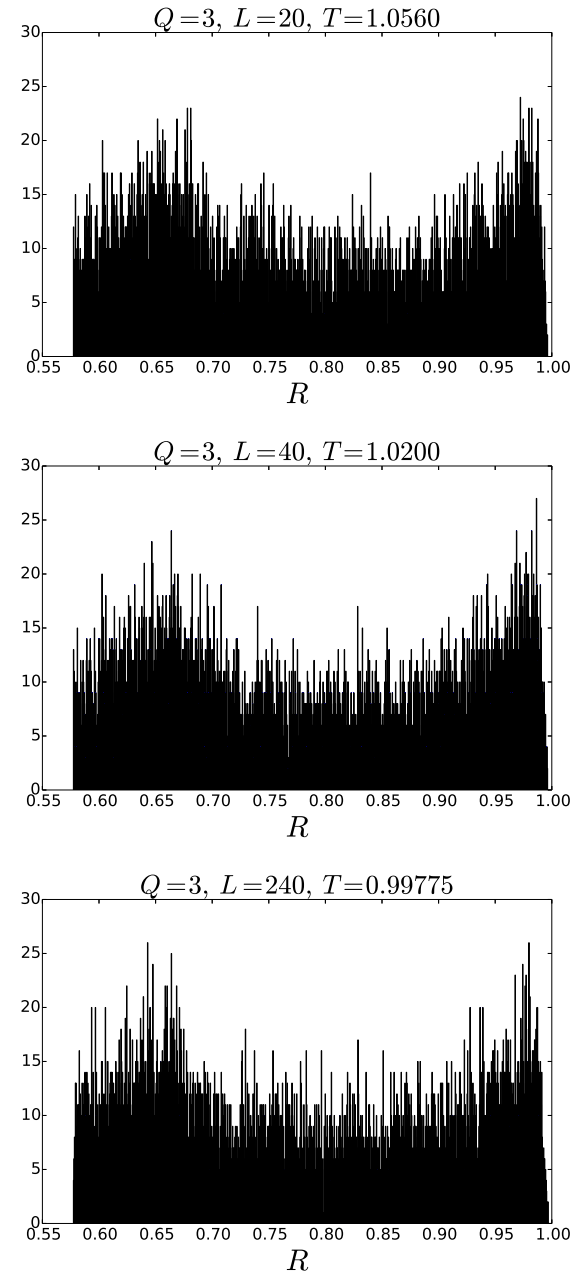

FIG. 8. Histograms of $R$ for $L=20$ (top), 40 (middle), and 240 (bottom) for 2D 3-states Potts model. Around ten thousand data close to the corresponding $T_{c}$ are generated for each of the plots. Data are recorded once for every 2000 updates after the thermalization. The histograms are produced by the "hist" function of pylab 33 .

lating physical quantities, such as the critical exponents, of second order phase transitions.

In computer science like the artificial intelligence, the ideas behind designing algorithms are sophisticated so that the constructed algorithms can apply for computations in many different areas. Such a concept is wellsuited in majority of scientific fields including physics. Notice different physical systems have their own special characters. Hence constructing appropriate NN based on certain properties of the considered physical systems is also an efficient way to conduct the investigation. In particular, several professional ML packages and libraries like Keras and TensorFlow are publicly available 34 37. These ML packages and libraries can be easily adopted to study specific systems. Hence for systems with less complexity, the strategy considered here, namely designing the NN according to the characters of the investi- 

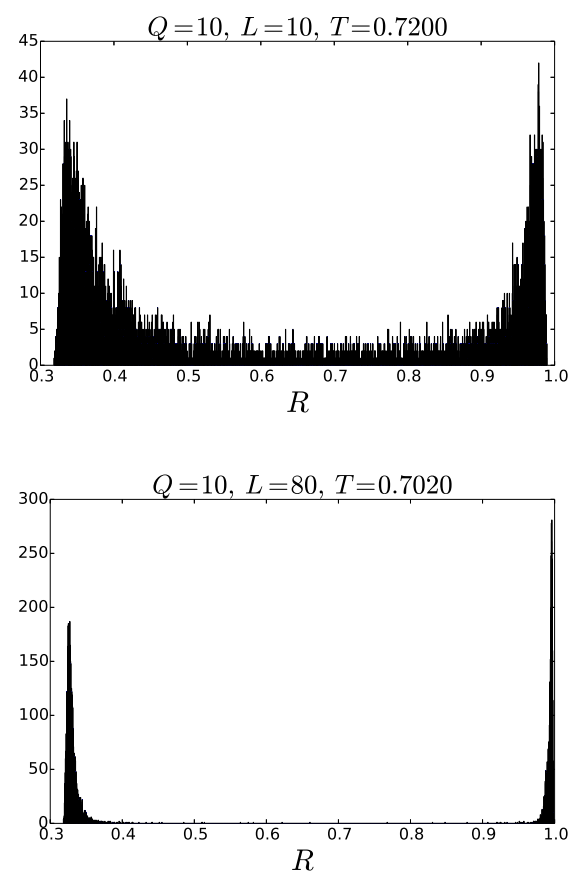

FIG. 9. Histograms of $R$ for $L=10$ (top) and 80 (bottom) for 2D 10-states Potts model. The testing sets for these results are the same as those used in fig. 6. The outcomes shown here are obtained using different convolutional kernel than that considered in fig. 6. The early stop criterion is removed in the calculations as well. The histograms are produced by the "hist" function of pylab

gated models is an effective approach as well. We would like to emphasize the fact that unlike other supervised NN used for studying the phase transitions of condensed matter systems, in our investigation no configurations of the disordered phase are in the pre-training set. This strategy greatly reduces the cost of the training process. Indeed, we have considered the situation of including the disordered configurations and using only two numbers as the outputs. The training for such trial calculations is much more demanding. In particular the related results are less accurate than those presented in the previous sections. Finally, we would like to point out as well that to determine $T_{c}$ using the ideas proposed in this study does not require any a priori information of $T_{c}$.

Notice a typical $\mathrm{NN}$ has several tunable parameters. For example, for the constructed NN here, the batch-size, the size of convolutional kernel, as well as the number of training objects are all adjustable variables. For the obtained results to be reliable, one has to make sure that the conclusions stay the same when the tunable parameters are reasonably varied. For instance, we have tested the results determined by using filter kernels with different sizes and matrix elements. In addition, We have also considered the situations of modifying the average pooling layer. Figure 9 shows the histograms of $R$ for $L=10$ and 80 for 10-states Potts model. In particular, in that calculations we use 3 by 3 convolutional kernel with each
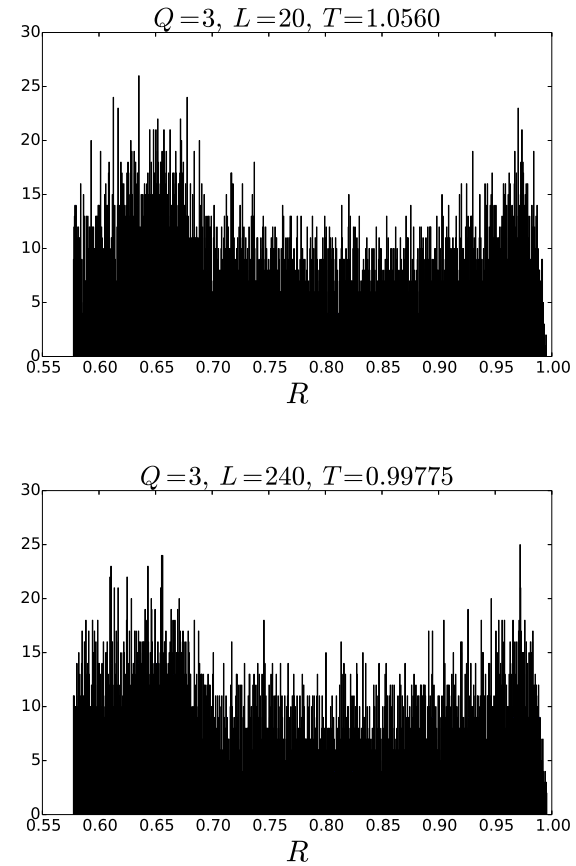

FIG. 10. Histograms of $R$ for $L=20$ (top) and 240 (bottom) for 2D 3-states Potts model. The testing sets for these results are the same as those used in figs. 7 and 8 The outcomes shown here are obtained using different convolutional kernel than that considered in fig. 7. The histograms are produced by the "hist" function of pylab

matrix element being randomly picked in $(-1 / 3,1 / 3)$. The early stop criterion is removed as well. The two peaks phenomena still remain quite visible in fig. 9. Similarly, for 3-states Potts model the histograms of $R$ for $L=20$ and 240 , obtained using 2 by 2 convolutional kernel, are shown in fig. 10. The results presented in fig. 10 lead to the same conclusion as that determined earlier, namely the phase transition of 3-states Potts model is second order.

In summary, based on the new analysis associated with the modified $\mathrm{NN}$, we find that while in the case of $L=240$ one may not be able to arrive at satisfactory outcomes $^{38}$, the resulting histograms of $R$ greatly favor the conclusions as those presented in previous sections regarding the nature of these considered phase transitions, provided that the tunable parameters are reasonably varied. In other words, the built NN is quite robust. Finally, we would like to emphasize the fact that although the conclusions regarding the nature of the considered phase transitions are unchanged when the used $\mathrm{NN}$ is modified, the corresponding $T_{c}(Q, L)$ at finite $L$ may slightly vary. This effect can be treated as one part of the errors for $T_{c}(Q, L)$ related to the $\mathrm{NN}$ methods. 


\section{ACKNOWLEDGEMENT}

Partial support from Ministry of Science and Technology of Taiwan is acknowledged. We thank D. Banerjee for reading the manuscript carefully and giving us very useful comments.
* fjjiang@ntnu.edu.tw

1 Trevor Hastie, Robert Tibshirani, and Jerome Friedman, The Elements of Statistical Learning: Data Mining, Inference, and Prediction, Second Edition (Springer, 2009).

2 Steven Horvath, Weighted Network Analysis: Applications in Genomics and Systems Biology (Springer Science+Business Media, LLC 2011).

${ }^{3}$ Laszlo Gyorfi, Gyorgy Ottucsak, and Harro Walk, Machine Learning For Financial Engineering (Imperial College Press, 2012).

4 M. Kircher, Martin Kircher, Daniela M Witten, Preti Jain, Brian J O'Roak, Gregory M Cooper, and Jay Shendure, Nature Genet. 46, 310315 (2014).

5 John D. Kelleher, Brian Mac Namee, and Aoife D'Arcy, Fundamentals of Machine Learning for Predictive Data Analytics: Algorithms, Worked Examples, and Case Studies (MIT Press, 2015).

${ }^{6}$ Maxwell W. Libbrecht and William Stafford Noble, Nature Reviews Genetics 16, 321332 (2015).

7 Ian Goodfellow and Yoshua Bengio, Deep Learning (Adaptive Computation and Machine Learning series) (MIT Press, 2016).

8 Adalbert F.X. Wilhelm and Hans A. Kestler, Analysis of Large and Complex Data (Studies in Classification, Data Analysis, and Knowledge Organization) (Springer International Publishing Switzerland, 2016).

9 S. Sachdev, Quantum Phase Transitions (Cambridge University Press, Cambridge, 1999).

10 A. W. Sandvik, AIP Conference Proceedings 1297 (2010).

11 A. Avella and F. Mancini, Strongly correlated Systems: Numerical Methods (Springer Berlin Heidelberg, 2013).

12 Juan Carrasquilla, Roger G. Melko, arXiv:1605.01735

13 Giuseppe Carleo, Matthias Troyer, Science 355, 602 (2017)

14 Giacomo Torlai and Roger G. Melko, Phys. Rev. B 94, 165134 (2016).

15 Peter Broecker, Juan Carrasquilla, Roger G. Melko, and Simon Trebst, arXiv:1608.07848.

16 Kelvin Ch'ng, Juan Carrasquilla, Roger G. Melko, and Ehsan Khatami, arXiv:1609.02552

17 Akinori Tanaka, Akio Tomiya, arXiv:1609.09087.

18 Evert P.L. van Nieuwenburg, Ye-Hua Liu, Sebastian D. Huber, arXiv:1610.02048.
19 Junwei Liu, Huitao Shen, Yang Qi, Zi Yang Meng, Liang Fu, arXiv:1611.09364.

20 Xiao Yan Xu, Yang Qi, Junwei Liu, Liang Fu, Zi Yang Meng, arXiv:1612.03804.

21 Lei Wang, Phys. Rev. B 94, 195105 (2016)

${ }^{22}$ Li Huang and Lei Wang, Phys. Rev. B 95, 035105 (2017).

23 Junwei Liu, Yang Qi, Zi Yang Meng, Liang Fu, Phys. Rev. B 95, 041101 (2017).

${ }^{24}$ Qianshi Wei, Roger G. Melko, Jeff Z. Y. Chen, arXiv:1701.04390

25 Recently, it was demonstrated in arXiv:1704.00080 that the unsupervised $\mathrm{NN}$ is capable of distinguishing first order phase transitions from second order ones. In this work we will focus on supervised NN.

${ }^{26}$ K. Binder, Journal of Statistical Physics 24(1), 69-86 (1981).

27 F. Y. Wu, Rev. Mod. Phys. 54, 235 (1982).

28 Alain Billoire, arXiv:hep-lat/9501003

29 R. H. Swendsen, and J.-S. Wang, (1987), Phys. Rev. Lett. $\mathbf{5 8 ( 2 )}, 86$ (1987).

30 M. E. Newman and G. T. Barkema, Monte Carlo Methods in Statistical Physics (Clarendon Press, 1999).

31 David P. Landau and Kurt Binder, A Guide to Monte Carlo Simulations in Statistical Physics (Cambridge University Press, 2014).

32 In this study, we do not carry out a detailed error analysis since very fine histograms of the relevant observables are used for the investigation.

33 All the histograms shown in this study are produced by the "hist" function of pylab. For better visualization, each of the histograms presented here may be obtained using different number of bins.

34 https://keras.io

35 http://scikit-learn.org/stable/

36 https://www.tensorflow.org

37 http://deeplearning.net/software/theano/

38 Occasionally one would arrive at unsatisfactory results for $L=240$ with the NN shown in fig. 4. 